\title{
Formas de intertextualidad en Il cavaliere e la morte de Leonardo Sciascia
}

\author{
Javier Serrano Puche \\ Universidad de Navarra \\ jserrano@unav.es
}

\section{Resumen}

El artículo examina la variedad y abundancia de referencias intertextuales sobre las que se construye el relato de la novela Il cavaliere e la morte (1988) de Leonardo Sciascia y que reflejan la importancia concedida por el escritor siciliano al valor veritativo de la escritura literaria. La apelación a libros precedentes del propio autor, a obras de otros escritores e incluso de otras disciplinas artísticas tejen un discurso polifónico que reúne los motivos temáticos y estilísticos más representativos del universo poético sciasciano.

Palabras clave: Leonardo Sciascia, intertextualidad, écfrasis, verdad literaria.

\section{Abstract}

The article examines the variety and abundance of intertextual references which constitutes the cornerstone upon which Sciascia builds up the story of the novel «The Knight and Death» (1988). These elements reflect the relevance given by the Sicilian author to the true value of the literary writing. The constant appeal to his former novels as well as some other writers' works, and the references to other artistic disciplines create a great polyphony in the author's discourse by which Sciascia reaches to gather the principal thematic and stylistic motives of his own poetic universe.

Key words: Leonardo Sciascia, intertextuality, ecphrasis, literary truth.

\section{Introducción}

Leonardo Sciascia tenía 67 años cuando escribió en el verano de 1988 Il cavaliere e la morte, publicada por la editorial Adelphi en aquel otoño. Fue una obra de madurez, escrita por el autor siciliano un año antes de morir y cuando ya era consciente de la cercanía de su muerte, no tanto por lo avanzado de su edad como por la grave enfermedad que padecía. ${ }^{1}$

1. Sciascia había visitado médicos de Palermo, Roma, Milán, París y Suiza, que atribuían su delicado estado de salud a las secuelas de la fractura de vértebra sufrida por el escritor en una caída en 1980 y a su condición de fumador empedernido, pero no conseguían enunciar un dia- 
No en vano, en una de las entrevistas concedidas con motivo de la publicación del giallo, Sciascia presentaba la novela como su última palabra sobre las realidades fundamentales:

Anche se continuerò a scrivere questo per me è un libro che chiude. Chiude quella che è la mia esperienza di vita, il mio giudizio sull'esistenza, sulle cose italiane, sul senso dell'essere vivi e sul senso della morte. È vero, sono serenamente disperato. Penso che nulla cambierà in Italia, almeno nell'arco della mia breve vita. $^{2}$

En gran medida, pronunciaba esa última palabra por boca del protagonista de la novela (el Vice, un subcomisario de policía que investiga el asesinato de un poderoso abogado y político), erigido en alter ego del autor por medio de diversas particularidades que evidenciaban que en su caracterización había claras connotaciones autobiográficas. También está gravemente enfermo $-\mathrm{y}$ de ahí que reflexione sobre el sufrimiento, sobre la vida que abandona y la muerte a la que se encamina-, y además comparte las preferencias literarias de Sciascia, su visión irónica de la realidad, la afición por el coleccionismo artístico o la adicción al tabaco. ${ }^{3}$

Todo esto le confería al texto un carácter de meditación in limine mortis que no pasó inadvertido ante la crítica literaria, que calificó la obra como «testamento laico» o «sonata fúnebre» por el modo con que «nello schema lucido della detective-story si è introdotto il pathos del dolore umano, del malessere esistenziale». ${ }^{4}$

En Il cavaliere e la morte aparecen las constantes formales y temáticas más representativas del universo poético sciasciano: la opción por el subgénero policiaco, cultivado de manera paródica y pirandelliana; la descripción de la realidad desde una mirada irónica, apoyada en la razón aunque abierta al misterio trascendente; la reflexión sobre la naturaleza del poder y su ejercicio en un contexto de corrupción política, dentro del cual el individuo honrado está

gnóstico claro. Los diversos medicamentos y terapias a los que se sometía el escritor apenas mitigaban su dolor. No será hasta abril de 1989 cuando, tras numerosos análisis en la clínica Città di Milano, finalmente le diagnostiquen un mieloma micromolecular, un raro tumor en la médula ósea que en la jerga médica llaman enfermedad de las «cadenas ligeras» y que causará la muerte del escritor el 20 de noviembre de 1989. Cfr. Matteo Collura, Sciascia. El maestro de Regalpetra, Madrid: Alfaguara, 2001, p. 355.

2. Leonardo SCIASCIA, "Scherzando con la vita», entrevista realizada por Daniela PASTI, Il Venerdì di Repubblica, 2 de diciembre de 1988. Citado por Olivia Barbella, Sciascia, Palermo: Palumbo, 1999, p. 86.

3. El propio Sciascia reconocía las semejanzas con su personaje: «Tutti i personaggi di un libro, oserei dire anche i più ignobili, hanno qualcosa in comune con l'autore (...) Ma, ovviamente, ci sono dei personaggi che rappresentano il suo prossimo più prossimo, in cui quasi si identifica. E così è per me il Vice di questo racconto. Mi somiglia in tante cose: inevitabilmente», Leonardo SCIASCIA, "Quel pasticciaccio dell'ottantanove», entrevista realizada por Rita CiRIO, L'Espresso, 11 de diciembre de 1988.

4. Natale Tedesco, «Un testamento laico», L'Ora, 19 de diciembre de 1989. 
condenado al aislamiento y al fracaso, pero reafirma su dignidad moral; la influencia de la idisoncrasia siciliana en el conjunto del relato; la recurrencia de la digresión y la citación literaria, etcétera. Todo ello confluye y se ensambla acaso en el modo artístico más logrado de esa extensa y variada producción literaria que se cimentó en 1956 con Le parrocchie di Regalpetra.

En la novela también está expresado un aspecto nuclear de la cosmovisión de Leonardo Sciascia que radica en su convicción expresa de que «la letteratura (...) è la più assoluta forma che la verità possa assumere». Esta identificación de la plena realización de la verdad con la escritura literaria se manifiesta especialmente a través de un variado repertorio de recursos intertextuales. En primer lugar, la citación de otras obras literarias, fundamentalmente a cargo del personaje protagonista, que sostiene su visión del mundo sobre una cultura libresca y artística, a través de la cual la realidad circundante se abre a su sentido profundo y verdadero. En segundo lugar, las alusiones interdisciplinares que constituyen al giallo como texto ecfrástico, ya que el protagonista del relato tiene en su despacho una copia del grabado de Durero El caballero, la muerte y el diablo (1513), que le inspira profundas reflexiones y del que la novela, por medio de una paráfrasis, toma su título. Por último, dado el carácter epilogal de Il cavaliere e la morte respecto del conjunto de la obra de Sciascia, pueden hallarse referencias intertextuales que ya aparecían en escritos precedentes del autor.

\section{Citación explícita e implícita de obras literarias}

En Il cavaliere e la morte la citación es un privilegio del que participan el narrador y dos personajes concretos: el Vice y la señora De Matis. Como ya señaló Ricciarda Ricorda en 1977 en el ensayo «Sciascia ovvero la retorica della citazione», en las obras del escritor siciliano hay personajes que ascienden al plano de la meditación, mientras que otros son rigurosamente excluidos; precisamente porque en el juego de las citaciones «alcuni personaggi acquistano la loro consistenza e si qualificano quiali portatori di un discorso essenziale per determinare la posizione ideologica di Sciascia stesso". ${ }^{6}$

En este sentido, el Vice y la señora De Matis tienen la capacidad de la citación literaria y de la divagación porque se constituyen como los portadores del discurso que compartiría el autor; de manera que, como veremos más adelante, también a través de ellos Sciascia repite algunas consideraciones que ya había expresado en entrevistas, ensayos u obras anteriores.

En el caso del protagonista, la citación a menudo no se exterioriza como acto de habla, sino que pertenece al discurrir de sus pensamientos, que el lector conoce por medio de la focalización interna. El acceso a su mente se hace 
respetando su propio vocabulario y omitiendo algunas acotaciones, pero a través de la tercera persona y el pretérito, por lo que cabría hablar de un modo de discurso indirecto libre.

La memoria del Vice fluye, divaga, establece asociaciones y vínculos entre elementos diversos, teniendo como catalizadora la literatura ${ }^{7}$ y como punto de partida los lugares y situaciones en los que él se encuentra, las imágenes, los objetos, las palabras. Por ejemplo, después de conversar con la señora Zorni, pasea por la ciudad y la visión de unos soportales le recuerda un verso de Dino Campana, que es parafraseado en función de la intención expresiva del protagonista: «Gli piacevano i portici, il camminarvi ozieggiando. Nell' isola dov'era nato, non c'era città che ne avesse. Gli archi fanno il cielo più bello, dice il poeta. I portici fanno più civili le città?». ${ }^{8}$ También mientras divaga paseando por el parque, adapta una pregunta retórica de Séneca para formular su temor ante la proliferación de los perros: «Si allontanò facendo ora attenzione ai tanti cani che andavano per il parco, gli venne da contarli. Tanti cani, forse più dei tanti bambini. E se gli schiavi si contassero?, si era domandato Seneca. E se si contassero i cani?»?

No siempre es necesario recurrir a la paráfrasis, pues en ocasiones hay una total identificación entre las palabras literarias y aquello que piensa el protagonista. Cuando visita la casa de su antigua amante y ella le abre la puerta en camisón, le viene a la memoria el título de una comedia de George Feydeau que expresa lo que en ese momento el Vice quisiera advertir a ella:

Lei venne ad aprirgli, come d'abitudine, dopo qualche minuto, in vestaglia: indossata, sapeva, in quel momento, per venire ad aprire la porta. Non andartene in giro tutta nuda. Ricordò, di molti anni prima, in un piccolo teatro di Roma (...) Franca Rame camminare per la scena non nuda, ma in non trasparente camicia da notte (...) In casa, lei se ne andava in giro tutta nuda. Cosa che, indubbiamente, come nella commedia di Feydeau, deliziava i dirimpettai, ma a lui aveva dato momenti di divampanti gelosia. Dentro di sé, ora, ne ride-

7. En Il cavaliere e la morte sucesos y actitudes en la vida de determinados escritores permiten interpretar un sentimiento del protagonista o servirle como orientación para sus decisiones últimas. Su frustración por la imposibilidad de que un médico pueda hacerse cargo del dolor exacto del paciente, aunque éste lo refiera con total precisión, encuentra un antecedente en una anécdota de Marcel Proust (cfr. Leonardo SCIASCIA, Il cavaliere e la morte, en Opere (1984-1989), Milano: Bompiani, 2002, p. 454), mientras que el ars moriendi de Michel de Montaigne se erige como modelo deseado para su propia vida y, además, sirve para que vuelva a tener estima por los perros: cfr. Ibid., p. 462. Sobre la opinión del ensayista francés acerca del modo de morir cfr. Michel de MONTAIGNE, «De la soledad», Ensayos completos, Madrid: Cátedra, 2003, p. 263-273.

8. Leonardo SCIASCIA, Il cavaliere e la morte, cit., p. 436. El verso de Campana pertenece a la primera de las Quattro poesie per Sibilla Aleramo: «I piloni fanno il fiume più bello / e gli archi fanno il cielo più bello / Negli archi la tua figura / Più pura nell'azzurro è la luce d'argento / Più bella la tua figura / Più bella la luce d'argento nell'ombra degli archi / Più bella della bionda Cerere la tua figura».

9. Leonardo SCIASCIA, Il cavaliere e la morte, cit., p. 461. 
va: e perciò gli venne alla memoria una scenetta (ancora il teatro) dei fratelli De Rege. ${ }^{10}$

Acordarse de los celos que en él causaba que ella anduviese desnuda por la casa, para deleite de los vecinos, le lleva a su vez a rememorar otra obra de teatro donde se desarrolla una escena cómica en torno al equívoco que provoca la palabra celosía.

Puede suceder, por otra parte, que el protagonista se sirva de un fragmento literario que se adecua totalmente a su pensamiento en una determinada situación, aunque difieran el contexto y los destinatarios respecto a aquellos de la obra original. Así, una frase de Hamlet referida a la cosmética femenina es aplicada a la «masa indigna» que está invadiendo el mundo y contra la cual se manifiesta el Vice:

Ancora bella, la vita; ma per chi ancora ne era degno. Se ne sentì non indegno, e come premiato. C'era da gridare: «Iddio vi ha dato un volto, voi ve ne fate un altro», non come Amleto alle donne, ai loro belletti, manteche e smalti, ma a tutti gli indegni, indegna massa di cui il mondo andava gremendosi; di gridarlo al mondo che andava assumendo questa sostanza: di essere indegno della vita. ${ }^{11}$

También los objetos inspiran el pensamiento y la memoria, ayudando a recorrer el pasaje que conduce de la realidad a la literatura, para luego retornar enriquecido a aquella. El Vice recibe de su médico una caja de morfina, pero él intenta aplazar su consumo, de igual manera que cree recordar ${ }^{12}$ que en La muerte de Iván Ilich, pese a ser conocida en la época, Tolstói no alude en el relato a la morfina. ${ }^{13}$

En otras ocasiones no son los objetos sino las palabras pronunciadas o pensadas por el Vice las que, por su semejanza con otras pertenecientes a obras literarias, le llevan a que se acuerde de éstas. Precisamente una reflexión sobre el sufrimiento y el modo de aplacarlo con la morfina, a través de la metáfora de

10. Ibid., p. 455.

11. Ibid., p. 460. Hamlet pronuncia la frase en un diálogo con Ofelia, en la primera escena del Acto tercero: cfr. William SHAKESPEARE, Hamlet, en Obras completas, tomo II, Madrid: Aguilar, 1974, p. 250.

12. En este punto concreto, al protagonista - y no sabemos si al propio Sciascia - le falla la memoria, pues sí se puede leer en La muerte de Iván Ilitch que «le daban opio y le ponían inyecciones de morfina». También en otro momento Iván Ilitch piensa: «iSi me inyectasen por lo menos morfina! ¡Me olvidaría entonces!»; Lev TOLSTOI, La muerte de Iván Ilitch, Barcelona: Editorial Juventud, 1966, p. 104 y 117.

13. «E gli venne un curioso pensiero, propriamente di curiosità: se nell'anno in cui Tolstoj raccontava la morte di Ivan Il'ic, la morfina, per quell'uso, la si conosceva già. 1885, 1886? C'era da credere la si conoscesse. Ma se ne faceva cenno, nel racconto? Gli pareva di no. E ne ebbe come un conforto: Tolstoj aveva allontanata la morfina dal suo personaggio forse per lo stesso suo sentimento. E pensando a quel racconto, cominciò a cercarne dentro di sé i riscontri», Leonardo SCIASCIA, Il cavaliere e la morte, cit., p. 453. 
la calma y la tormenta, se encadena con el título de un poema de Giacomo Leopardi, al que le siguen otros tres también pertenecientes a sus Canti y que para el Vice están asociados a la infancia y la escuela:

Belli gli effetti della morfina, più se succedevano all'insopportabile sofferenza. Più forte la tempesta, più dolce poi la quiete. La quiete dopo la tempesta, Il sabato del villaggio, Il passero solitario, Linfinito: quali grandi e profondi sentimenti, con assoluta semplicità, e magari con immagini banali, quel poeta di felice infelicità aveva rivelato e indelebilmente impresso nella memoria degli italiani che si potevano ora dir vecchi. ${ }^{14}$

Por su parte, la contemplación de la figura de la muerte en el grabado de Durero, por la imagen de mendicidad que transmite, suscita el recuerdo de unos versos de Ungaretti, incluidos en el relato sin más preámbulo ni marca textual que los distinga salvo su entrecomillado: «E la Morte, nonostante i minacciosi orpelli delle serpi e della clessidra, era espressiva più di mendicità che di trionfo. "La morte si sconta vivendo". Mendicante, la si mendica». ${ }^{15}$

Otra muestra de este modo de rememoración a través de las palabras es la conversación con el Grande Giornalista, a la que el protagonista pone fin diciendo «Sono già sbarcato su un'isola deserta». Una frase que le provoca al Vice el deseo de alcanzar esa isla desierta, que para él está encarnada en aquella que da título a la novela de aventuras de Robert Louis Stevenson:

Poteva dunque, dall'ultima battuta di quel colloquio, vagheggiare l'isola deserta, come su una mappa svolgervi antico sogno, antica memoria; per quanto antiche gli erano diventate certe cose dell'infanzia, dell'adolescenza. L'isola del tesoro: una lettura, aveva detto qualcuno, che era quanto di più si poteva assomigliare alla felicità. Pensò: stasera lo rileggerò (...) Finì di rileggersi Lisola del tesoro: qualcosa, ancora, che somigliava alla felicità. ${ }^{16}$

De igual manera que antes veíamos que el verso «Gli archi fanno più bello il cielo» se atribuye al "poeta», sin especificar que es de Dino Campana o que no se señala a Ungaretti como autor de aquel «La morte si sconta vivendo», aquí no se revela que ese "qualcuno" que escribió que la lectura de La isla del tesoro es «lo más parecido a la felicidad» fue Borges. ${ }^{17}$ Esta modalidad de intertextualidad sugerida, que no explicita sus fuentes originales, convierte la lectura de Il cavaliere e la morte en un ejercicio de hermenéutica y de aceptación del

14. Ibid., p. 452.

15. Ibid., p. 449. Los versos de Ungaretti son del poema Sono una creatura, incluido en Il porto sepolto (1916): "Come questa pietra / del S. Michele / così fredda / così dura / così prosciugata / così refrattaria / così totalmente / disanimata / Come questa pietra / è il mio pianto / che non si vede / La morte / si sconta / vivendo", Giuseppe UNGareTTI, Vita d'un uomo: poesie, vol. L'allegria, Verona: Arnoldo Mondadori, 1970, p. 60.

16. Leonardo SCIASCIA, Il cavaliere e la morte, cit., p. 448 y 463.

17. Cfr. Jorge Luis BORGES, «La fruición literaria», en El idioma de los argentinos, Madrid: Alianza, 1998, p. 91-98. 
juego intelectual propuesto por el autor, y confiere al texto un discurso polifónico y abierto a la reinterpretación.

Una muestra de ello está, dentro de su extensa reflexión del penúltimo capítulo, cuando el protagonista compara su infancia con la de los niños que ve jugar en el parque y les augura un futuro sin alegría ni fantasía. Entre otras razones porque, dado que la memoria es un elemento dinámico y tan importante para la dignidad del hombre, el Vice teme que desde las instancias del poder corrupto se intentará impedir su desarrollo libre y con ello los ejercicios que la hacen dúctil, sutil, como la obligación escolar de memorizar algunos poemas; práctica que, no obstante, él recuerda con afecto:

Erano ancora capaci di gioia, di fantasia: ma li aspettava una scuola senza gioia e senza fantasia, la televisione, il computer, l'automobile da casa a scuola e da scuola a casa, il cibo ricco ma dall'indifferenziato sapore di carta assorbente. Non più, nella memoria, la tavola pitagorica, "La donzelletta vien dalla campagna...», «Scendeva dalla soglia...», «I cipressi che a Bolghieri...»: sevizie del passato. La memoria era da abolire, la Memoria; e quindi anche quegli esercizi che la rendevano duttile, sottile, prensile. ${ }^{18}$

Cabe advertir de nuevo en el fragmento anterior la intertextualidad velada de Sciascia. En este caso, en la omisión del nombre del autor y de la obra a la que pertenece cada una de las tres frases incluidas en el relato como ejemplos de lo que el protagonista memorizaba en sus años de infancia. Es un recuerdo íntimo, desarrollado en el flujo de conciencia del Vice y por eso asume esa forma fragmentaria e incompleta, que para su correcta interpretación requiere el esfuerzo y competencia de un lector que sepa identificar el comienzo de Il sabato del villaggio de Leopardi: "La donzelletta vien dalla campagna...»; «Scendeva dalla soglia...» como parte del capítulo 34 de I promessi sposi de Manzoni (en el que Renzo atraviesa una Milán arrasada por la peste); y que "I cipressi che a Bolghieri...» es el primer verso del poema Davanti San Guido de Giosuè Carducci. ${ }^{19}$

Ya en el capítulo precedente el Vice había aludido a las pruebas escolares de memorización de poemas y también entonces había ejercitado la memoria como antaño, dejando constancia textual con la inclusión de un par de fragmentos de poesías de Victor Hugo:

Lo si leggeva ancora [a Leopardi], nella scuola? Forse ancora; ma certo nessun ragazzo ne sapeva i versi a memoria. Par coeur, come diceva la professoressa di francese quando assegnava le poesie di Victor Hugo, quasi sempre di Victor Hugo. Le ricordava ancora: «Devant la blanche ferme où parfois vers midi Un vieillard vient s'asseoir sur le seuil attiédi...»; «Oh! combien de marins, combiens

19. Cfr. Giacomo Leopard, I canti, Milano: Bietti, 1933, p. 113; Alessandro Manzoni, I promesi spossi, en Opere, Milano: Ugo Mursia, 1964., p. 716; Giosuè CarduCCI, Antologia carducciana: poesie e prose, Bologna: Nicola Zanichelli, 1936, p. 104. 
de capitaines Qui sont partis joyeux pour des courses lointaines, Dans ce morne horizon se sont évanouis...», e questa più par coeur, ormai. Bellissima espressione; e la traduceva «nel cuore, dal cuore, per il cuore». ${ }^{20}$

Aunque haga referencia al autor francés, la ausencia de los títulos de los poemas (La Vache y Oceano Nox, respectivamente) y su transcripción en la lengua original preservan ese tono privado y misterioso de la citación; de tal modo que, aunque todo lector es invitado a participar de ella, probablemente sólo sea identificada y comprendida por unos pocos. De ahí que no parezca injustificado señalar que, como su admirado Stendhal, a esas alturas de la vida Sciascia escribe su testamento literario "to the happy few». ${ }^{21}$

Por otra parte y como resulta evidente por los ejemplos que hemos señalado, en la citación intertextual atribuible por medio de la focalización interna al Vice existe una fuerte vinculación entre la memoria y la literatura, asociadas desde la perspectiva de la afectividad. Vinculación que queda reforzada cuando el protagonista, al reflexionar sobre el sentido destructivo del dolor, señala que hay momentos en que cae sobre «ogni piacere ancora possibile, sull'amore, sulle pagine amate, sui lieti ricordi». ${ }^{22}$ Esta noción del acto de recordar como una forma de amor - que se materializa en esa expresión ("par coeur») con que la profesora de francés anunciaba el reparto de los poemas - remite a Alberto Savinio, que en la voz «Memoria» de su Nuova enciclopedia defendía esa misma identificación. ${ }^{23}$ También podemos atribuir en buena medida a la influencia saviniana la atención de Sciascia a la palabras, que ponen en marcha su fantasía y su intelecto y que, como hemos podido comprobar, son el origen de una gran parte de la citación intertextual presente en Il cavaliere e la morte.

En el relato policiaco también hay, sin llegar a constituirse como citas intertextuales explícitas, alusiones más o menos directas a obras de escritores que sin duda eran conocidos y estimados por Sciascia. Entre los autores que son objeto de citación implícita está Antonio Machado, al que el autor siciliano consideraba «il più puro poeta di Spagna» ${ }^{24} \mathrm{y}$ del que parafrasea dos versos cuando el protagonista del giallo piensa que los perros que encuentra por el parque parecen soñar con «boschi irti ed oscuri, pietraie impervie»; ${ }^{25}$ en referencia a unos versos de Campos de Castilla: «en torno a Soria, obscuros encinares, / ariscos pedregales». ${ }^{26}$ Además, parece claro que hay un eco de la

20. Leonardo Sciascia, Il cavaliere e la morte, cit., p. 452. Cfr. Victor HugO, Oeuvres poétiques: Tome 1. Avant l'exil: 1802-1851, París: Gallimard, 1992, p. 969 y 1116.

21. Es la dedicatoria con la que Stendhal concluye La Chartreuse de Parme y que expresa su convicción de que escribe "a unos pocos felices», pues profetizaba que la llegada de sus verdaderos lectores no se produciría hasta 1880 y, después, alrededor de 1935.

22. Leonardo SCIASCIA, Il cavaliere e la morte, cit., p. 454.

23. Cfr. Alberto Savinio, Nuova enciclopedia, Milano: Adelphi, 1997, p. 257, s.v. Memoria.

24. Leonardo SCIASCIA, Ore di Spagna, Milano: Bompiani, 2000, p. 73.

25. ID., Il cavaliere e la morte, cit., p. 462.

26. Antonio Machado, Campos de Soria, VII, de Campos de Castilla, en ID., Poesía y prosa, edición crítica de Oreste Macrì, Tomo II, Madrid: Espasa-Calpe, 1989, p. 515. 
imagen poética del segundo de los "Consejos» de Machado («Moneda que está en la mano / quizá se deba guardar; / la monedita del alma / se pierde si no se da») ${ }^{27}$ en una metáfora pesimista desarrollada por el Vice que participa del mismo campo semántico: «Forse tutto nel mondo stava accadendo a somiglianza dell'inflazione, la moneta del vivere ogni giorno perdeva di valore; la vita intera era una specie di vacua euforia monetaria senza più alcun potere di acquisto". ${ }^{28}$

También en su primera reflexión ante el grabado El caballero, la muerte y el diablo realiza una descripción del dibujo ("La morte; e quel castello lassù, irraggiungibile» $)^{29}$ que puede leerse como una referencia alusiva a El castillo de Franz Kafka; pues, si en la incisión dureriana el castillo está allá y arriba (lassù), la misma disposición geográfica marca el inicio de la novela kafkiana: «Allí arriba, el castillo, curiosamente oscuro ya, al que K. había esperado llegar aquel día, volvía a alejarse». ${ }^{30}$

En el giallo, el recinto fortificado es visto por el Vice como «chiusa cittadella in alto, la cittadella della suprema verità, della suprema menzogna»; 31 de manera que, al ser inalcanzables tanto una como otra, el caballero de Durero ha de moverse en la incertidumbre gnoseológica. De igual modo, la actuación de K. está marcada por la falta de certezas. No sabe si logrará llegar al castillo y, además, como ha señalado Roberto Calasso

no queda del todo claro lo que sucedió antes del «largo, difícil viaje» que lo condujo hacia el Castillo. ¿Recibió una convocatoria, o bien emprendió el viaje precisamente para obtenerla? No hay modo de saberlo con certeza. En cambio, existen muchas maneras de agravar y exasperar la incertidumbre. ${ }^{32}$

Las referencias veladas a Machado y Kafka no son las únicas que pueden hallarse en Il cavaliere e la morte. En este mismo sentido, no parece infundado aventurar — dada la admiración y el conocimiento que tenía Sciascia de I promessi sposi- que, cuando describe la aversión del Vice por la práctica policial de realizar los arrestos en mitad de la noche, el escritor siciliano se haya acordado de aquel que sufre Renzo en Milán, ${ }^{33}$ pues se desarrolla de modo muy

27. ID., Consejos, II, de Soledades, en ID., Poesía y prosa, p. 470.

28. Leonardo SCIASCIA, Il cavaliere e la morte, cit., p. 454.

29. ID., Il cavaliere e la morte, cit., p. 407.

30. Franz KafKA, El castillo, en Obras completas, vol. 1. Novelas, Barcelona: Galaxia Gutenberg-Círculo de Lectores, 1999, p. 706.

31. Leonardo SCIASCIA, Il cavaliere e la morte, cit., p. 450.

32. Roberto Calasso, K., Barcelona: Anagrama, 2005, p. 14.

33. "Allo spuntar del giorno, Renzo russava da circa sett'ore, ed era ancora, poveretto! sul più bello, quando due forti scosse alle braccia, e una voce che dappiè del letto gridava: - Lorenzo Tramaglino!- - lo fecero riscotere. Si risentì, ritirò le braccia, aprì gli occhi a stento; e vide ritto appiè del letto un uomo vestito di nero, e due armati, uno di qua, uno di là del capezzale. E, tra la sorpresa, e il non esser desto bene, e la spranghetta di quel vino che sapete, rimase un momento come incantato; e credendo di sognare, e non piacendogli quel sogno, si dimenava, come per isvegliarsi affatto", Alessandro MANZONI, op. cit., p. 453. 
similar a "quell'aprirsi a spiraglio della porta, quegli occhi di sonno e di diffidenza che guatavano; la violenta spinta alla porta, l'irruzione; e dentro, poi, lo svegliarsi agitato». ${ }^{34}$ También su posterior paseo por las calles, custodiado por los alguaciles y entre la turbamulta, ${ }^{35}$ parece estar implícitamente referido en el giallo de Sciascia en la detención de un presunto terrorista del grupo «i figli dell'ottantanove» en una cabina telefónica y en su traslado a comisaría seguido por una multitud exaltada:

Lo condusse al posto di polizia più vicino; che non era però tanto vicino, sicché alla folla che andava formandosi dietro - un corteo prima di arrivare a destinazione- più volte fu costretto a dichiarare che si trattava di un presunto figlio dell'ottantanove (...) Al che ad un certo punto, dal vociare della folla dietro, gli avvenne di sudar freddo per la paura che si volesse far giustizia svelta contro la lenta giustizia. ${ }^{36}$

Por otra parte, el origen de la investigación policial relatada en Il cavaliere e la morte guarda una clara similitud con el hecho desencadenante de La broma, una novela de Milan Kundera que había recibido los elogios del escritor siciliano en una de las entradas de su diario Nero su nero (1979). ${ }^{37}$ En el giallo es una supuesta broma escrita por el empresario Cesare Aurispa la que le delata como sospechoso de haber llevado a cumplimiento la anotación "Ti ucciderò» dirigida al abogado Sandoz; y en la obra del autor checo el protagonista, Ludvik, escribe a modo de juego una postal con consignas en contra del régimen comunista, pero será interceptada y tomada en serio por el partido. ${ }^{38}$ Sin embargo, mientras que para Ludvik tendrá graves consecuencias para su futuro, pues vive en una sociedad que ha perdido el sentido del humor, Aurispa sabe que por su condición de potentado goza en la práctica de inmunidad ante la investigación policial y no teme, sea verdadero culpable o no, la identificación entre su expreso deseo y la muerte del abogado.

34. Leonardo SCIASCIA, Il cavaliere e la morte, cit., p. 410.

35. «Quelli guardarono più attentamente la comitiva, e si fermarono; con loro si fermarono altri che arrivavano; altri, che gli eran passati davanti, voltatisi al bisbiglìo, tornavano indietro, e facevan coda (...) Ahi! ahi! ahi! — grida il tormentato: al grido, la gente s'affolla intorno; n'accorre da ogni parte della strada: la comitiva si trova incagliata», Alessandro MANZONI, op. cit., p. 459.

36. Leonardo SCIASCIA, Il cavaliere e la morte, cit., p. 445.

37. Cfr. ID., Nero su nero, cit., p. 718.

38. «Para un trotskista el optimismo de los constructores del socialismo no es más que opio. Y tú eres trotskista. Por Dios, cómo se os ha ocurrido eso, me defendí. Lo has escrito tú ¿o no? Es posible que haya escrito algo por el estilo en broma, ya hace más de dos meses, no lo recuerdo. Te lo podemos recordar nosotros, dijeron y me leyeron mi postal. El optimismo es el opio del pueblo. ¡El espíritu sano hiede a idiotez! ¡Viva Trotsky! Ludvik. En la pequeña sala del secretariado político aquellas frases sonaban de un modo tan horrible que en ese momento sentí miedo y me di cuenta de que tenían un poder destructivo que yo no iba a ser capaz de resistir. Camaradas, era una broma, dije y sentí que nadie podría creerme», Milan KunderA, La broma, Barcelona: Seix Barral-Booket, 2006, p. 44. 


\section{Il cavaliere e la morte como texto ecfrástico}

Hasta ahora hemos examinado la variedad de formas que adopta la intertextualidad en Il cavaliere e la morte cuando esa relación se produce entre textos verbales. Sin embargo, en el giallo también debemos considerar otra modalidad que consiste en la alusión a un texto icónico, esto es, el grabado de Alberto Durero El caballero, la muerte y el diablo (Ritter, Tod und Teufel, 1513). ${ }^{39}$ Sobre él hay cuatro referencias directas ${ }^{40}$ a lo largo del relato, que hacen que su presencia e influencia abarque el conjunto de la historia narrada. De hecho, el relato comienza con una de esas referencias que, dada su extensión y contenido, sitúa desde entonces al grabado dureriano como un elemento poético consustancial a la obra de Sciascia:

Quando alzava gli occhi dalle carte, e meglio quando appoggiava la testa sull'orlo dell'alto e duro schienale, la vedeva nitida, in ogni particolare, in ogni segno, quasi il suo sguardo acquistasse un che di sottile e puntuto e il disegno rinascesse con la stessa precisione e meticolosità con cui, nell'anno 1513, Albrecht Dürer lo aveva inciso. L'aveva acquistata, molti anni prima, ad un'asta: per quell'improvviso e inconsulto desiderio di possesso che a volte lo assaliva di fronte a un quadro, una stampa, un libro. Laveva contesa agli altri che la volevano, arrivando quasi ad odiare il più tenace, che gliela aveva poi abbandonata ad un prezzo che, corrispondendo al suo stipendio di due mesi, al momento di pagarlo gli diede un certo sgomento. Ingente non soltando in rapporto alle sue possibilità; ma ora, per il vertiginoso crescere dell'inflazione e per il moltiplicato valore delle cose di Dürer e di ogni altro grande incisore, irrisorio. Se l'era portata dietro da una sede all'altra, da un ufficio all'altro; attaccandola sempre alla parete di fronte alla scrivania. Ma di tutti quelli che in tanti anni erano entrati nel suo ufficio, soltanto uno (un truffatore ingegnoso che giovialmente accettava la sorte che da quell'ufficio lo avrebbe per qualche anno fatto ospite di un carcere inospitale) si era soffermato a guardarla e ad apprezzarla: proprio ad apprezzarla, secondo i più recenti cataloghi dei mercanti di stampe zurighesi e parigini.

Quell'apprezzamento lo aveva un po'allarmato; in un soprassalto di grettezza, di avarizia, aveva deciso di portarsela a casa; ma se ne dimenticò subito. Si era ormai abituato ad averla di fronte, nelle tante ore d'ufficio. Il cavaliere, la morte e il diavolo. Dietro, sul cartone di protezione, c'erano i titoli, vergati a matita, in tedesco e in francese: Ritter, Tod und Teufel; Le chevalier, la mort et le diable. E misteriosamente: Christ? Savonarole? Il collezionista o il mercante che si era interrogato su quei nomi pensava forse che l'uno o l'altro Dürer avesse voluto simboleggiare nel cavaliere? ${ }^{41}$

39. Como señala Concha Huidobro, «en 1513 y 1514 Durero se dedica principalmente al grabado al buril y realiza sus tres mejores estampas en esta técnica: El caballero, La melancolía y San Jerónimo en su celda, conocidas como sus obras maestras en cobre (Die Meisterstiche) (...) Tienen la misma concepción, la misma técnica y el mismo formato. La mayoría de los autores piensa que describen tres temperamentos o modos de ver el mundo: el caballero cristiano que defiende la religión de manera activa, el santo sabio y espiritual y el genio creativo", Concha Huidobro, Durero grabador, Madrid: Electa, 1999, p. 35-36.

40. Cfr. Leonardo SCIASCIA, Il cavaliere e la morte, cit., p. 407-408, 449-450, 457 y 459.

41. Ibid., p. 407-408. 
Ya en el orden paratextual cabe advertir la importancia del grabado dureriano, por el título parafrásico del giallo y la reproducción de la obra gráfica en la portada del libro, al menos en su edición original y en la traducción española. Pero en Il cavaliere e la morte de Leonardo Sciascia, El caballero, la muerte y el diablo de Alberto Durero no sólo adquiere un sentido alegórico, sino que también es objeto de un ejercicio de écfrasis literaria, por cuanto que el relato ofrece una representación escrita de aquella representación visual. Como tal, es diversa de la que podría proporcionar la crítica de arte o la historia del arte, puesto que en éstas la écfrasis siempre se refiere a un obra plástica ya existente, y sólo tiene sentido si está basada en el análisis formal de su objeto, mientras que la écfrasis literaria presupone el cuadro, sea este real (como el caso que nos ocupa) o ficticio. No es la única diferencia, como expone Michel Riffaterre:

La écfrasis crítica formula juicios de valor variados y basados en principios estéticos explícitos, condena o elogia, quiere formar el gusto de sus lectores. Por el contrario, la écfrasis literaria busca su admiración: al ser una variedad del encomio, se convierte de hecho en un blasón de la obra pictórica. La écfrasis crítica tiene por objeto obras de arte que se bastan a sí mismas y cuyo valor puede ser independiente del contexto. La écfrasis literaria tiene por objeto esas mismas obras, pero también obras de arte reales o imaginarias insertadas en una obra literaria - por ejemplo, en una novela. Forman parte del decorado, o bien tienen una función simbólica, o pueden incluso motivar los actos y las emociones de los personajes. ${ }^{42}$

El grabado de Durero participa de cada una de estas tres características en la obra de Sciascia: decora el despacho del protagonista, su figura principal adquiere una doble valencia simbólica respecto al personaje de la novela a quien podría representar, como veremos más adelante, y, en último término, cabría otorgarle el carácter de emblema de la vida del Vice.

La écfrasis se inicia, por tanto, con el propio inicio del relato, aunque aún de modo aproximativo y atendiendo sobre todo a las circunstancias de su adquisición por el protagonista y a elementos contextuales que, no obstante, ya suscitan una disposición interpretativa frente a la obra artística. Junto a indicios que ayudan a situar al lector (el título del grabado, su autor y el año en que lo realizó, la técnica empleada) hay una anotación propiamente descriptiva del contenido del dibujo («La morte; e quel castello lassù, irraggiungibile») que, además de sintetizar el conflicto dramático allí narrado, resulta muy sugestiva no sólo por las connotaciones kafkianas ya apuntadas, sino también porque cabría leerla como una anticipación velada del destino que aguarda al Vice.

La naturaleza ecfrástica del relato de Sciascia se evidencia en mayor medida en el capítulo noveno, donde sí existe una evocación detallada de 
las tres figuras principales del grabado, que creemos conveniente citar íntegramente:

Stava intanto guardando Il cavaliere, la morte e il diavolo. Forse Ben Gunn, per come Stevenson lo descriveva, un po' somigliava alla Morte di Dürer; sicché gli parve prendesse, la Morte di Dürer, un riflesso di grottesco. L'aveva sempre un po' inquietato l'aspetto stanco della Morte, quasi volesse dire che stancamente, lentamente arrivava quando ormai della vita si era stanchi. Stanca la Morte, stanco il suo cavallo: altro che il cavallo del Trionfo della morte e di Guernica. E la Morte, nonostante i minacciosi orpelli delle serpi e della clessidra, era espressiva più di mendicità che di trionfo. "La morte si sconta vivendo». Mendicante, la si mendica. In quanto al diavolo, stanco anche lui, era troppo orribilmente diavolo per esser credibile. Gagliardo alibi, nella vita degli uomini, tanto che si stava in quel momento tentando di fargli riprendere il vigore perduto: teologiche terapie d'urto, rianimazioni filosofiche, pratiche parapsicologiche e metapsichiche. Ma il Diavolo era talmente stanco da lasciar tutto agli uomini, che sapevano fare meglio di lui. E il Cavaliere: dove andava così corazzato, così fermo, tirandosi dietro lo stanco Diavolo e negando obolo alla Morte? Sarebbe mai arrivato alla chiusa cittadela in alto, la cittadella della suprema verità, della suprema menzogna?

Cristo? Savonarola? Ma no, ma no. Dentro la sua corazza forse altro Dürer non aveva messo che la vera morte, il vero diavolo: ed era la vita che si credeva in sé sicura: per quell'armatura, per quelle armi. ${ }^{43}$

Podemos apreciar aún más el carácter literario de la écfrasis sciasciana si la comparamos con la descripción que del mismo grabado hace Erwin Panofsky, historiador del arte y experto en la obra de Durero. Él atribuye como fuente inspiradora para la caracterización del caballero el tratado Enchiridion Militis Christiani, publicado en 1504 por Erasmo de Rotterdam y que presenta el ideal del cristiano como un soldado-peregrino cuya fe es tan viril y serena que los peligros y tentaciones del mundo (la carne, el demonio y el mundo) pasan a ser simples espectros sin fuerza. ${ }^{44}$ Un ideal que es representado por el artista alemán en su obra, que es así descrita por Panofsky:

Este Jinete monumental (...) se sitúa ante un fondo de peñascos imponentes y árboles desnudos, con su punto de destino, la inexpugnable «fortaleza de la Virtud», todavía muy lejos, al final de un camino empinado y serpenteante. De la penumbra de este escenario «abrupto y temible» emergen las figuras de la Muerte y el Demonio (...) La Muerte lleva corona real y monta un jamelgo flaco y lánguido con un cencerro; pero ella es aún más espantosa porque no está representada como un esqueleto de verdad, sino como un cadáver en descomposición, con ojos tristes, sin labios ni nariz, con la cabeza y el cuello

43. Ibid., p. 449-450.

44. "Ten delante esta tercera regla si es que te horroriza el camino de la virtud, bien porque te puede parecer duro y triste, bien porque tendrás que enfrentarte a los tres enemigos más encarnizados: la carne, el demonio y el mundo. A ejemplo del Eneas de Virgilio, has de disipar esos trasgos y fantasmas que te asaltan en las mismas fauces del infierno», ERASMO, Enquiridion. Manual del caballero cristiano, Madrid: Biblioteca de Autores Cristianos, 1995, p. 127. 
ceñidos de serpientes. Se ha puesto a la altura del Jinete y vanamente trata de atemorizarle mostrándole un reloj de arena, en tanto que el Demonio, con hocico de cerdo, se le acerca por detrás armado de una pica. ${ }^{45}$

Al subrayar el aspecto grotesco de la Muerte y del Diablo, el historiador concuerda, por tanto, con la reflexión del personaje de ficción, aunque difieren las perspectivas, la finalidad y los elementos y recursos de que se sirven ambos para analizar la obra. Para el protagonista del giallo el recurso a la citación intertextual es indispensable en esa tarea interpretativa y por ello emplea la comparación con otros motivos literarios o artísticos para comprender mejor la obra del grabador germano. Como justo antes había reflexionado sobre los personajes de La isla del tesoro y dado que «la sua mente era così libera, così in vacanza e in vaganza», ${ }^{46}$ asocia con la figura de la Muerte a Ben Gunn —el marinero abandonado en la isla por sus compañeros tres años antes de que llegase allí la Hispaniola de John Silver-, por el aspecto ridículo y cansado que caracteriza a ambos. También es el rasgo distintivo del caballo de la Muerte y por ello el Vice emplea de nuevo la función comparativa, dando pie a una intertextualidad icónica, en este caso no en términos de identificación sino de diferenciación: el «jamelgo flaco y lánguido», en palabras de Panofsky, contrasta con los caballos vigorosos representados en el fresco medieval El triunfo de la muerte ${ }^{47}$ y en el Guernica de Picasso.

Esa imagen de mendicidad más que de triunfo, tanto de la Muerte como de su cabalgadura, no es atribuida según razones historiográficas como hacía aquel al relacionarla con el Enchiridion, sino que se interpreta desde una visión poética existencialista ( «l'aspetto stanco della Morte, quasi volesse dire che stancamente, lentamente arrivava quando ormai della vita si era stanchi»). De ahí que a continuación emerjan en la memoria del protagonista los versos de Ungaretti que sentencian que la muerte se va pagando con la vida. Igualmente ocurre en la percepción que tiene del Diablo. Allí donde Panofsky veía la representación de un espectro sin poder, debido a la fe inconmovible del caballero, el Vice piensa por el contrario que, precisamente por su inoperancia, el Diablo ha dejado su trabajo en manos de los hombres, que son más eficaces que él.

La divergencia entre las valoraciones de la écfrasis crítica y la écfrasis literaria se acentúa aún más en la interpretación de la figura principal, pues mientras que la primera la identifica con aquel ideal del soldado-peregrino cristiano del que escribió Erasmo, la segunda llega a cuestionarse si acaso Durero no había representado en ella «la vera morte, il vero diavolo», que era la vida convencida de que estaba a salvo dentro de aquella armadura.

45. Erwin Panofsky, Vida y arte de Alberto Durero, Madrid: Alianza, 1982, p. 168.

46. Leonardo SCIASCIA, Il cavaliere e la morte, cit., p. 449.

47. Aunque es un tema iconográfico habitual en la Edad Media, asociado con el de las «Danzas macabras» y cuya representación más conocida es la Pieter Brueghel el Viejo, la imagen de un caballo triunfante remite en concreto al fresco anónimo del siglo XV que se conserva en el Palazzo Abatellis de Palermo, lo cual concuerda por otra parte con el origen siciliano del Vice. 
A este respecto, aunque en la obra de Durero parecen cristalizar icónica y sincréticamente los confines del destino del Vice, ${ }^{48}$ no conviene dar por supuesto que el valor simbólico del caballero es unívoco y que sólo permite su interpretación en términos de identificación con el protagonista. De hecho, entre los estudiosos de mayor relieve de la obra sciasciana ha suscitado diversas interpretaciones, a las que conviene hacer referencia.

La tesis de que el caballero simbolice al protagonista del relato ha sido la más ampliamente desarrollada y apoyada; y a los elementos del texto que ayudan a sustentarla se suman algunos factores contextuales análogos, como la soledad del propio Leonardo Sciascia en la defensa de ciertos valores ante la opinión pública italiana o la enfermedad que padecía el autor mientras escribía la obra. De esta manera, el caballero sería un alter ego del Vice, que a su vez es un alter ego de Sciascia. Éste, a través del protagonista de la novela, llega a reflejarse en el protagonista del grabado. ${ }^{49}$

Massimo Onofri, basándose en el fragmento arriba seleccionado, concuerda con la hipótesis de una identificación del Vice con el caballero:

Quella "vita in sé sicura», che il Cavaliere nasconde dentro la corazza, infatti, non è altro che la vita degli uomini che se ne vanno impettiti e boriosi, quando rimuovono il pensiero della morte, forse la stessa vita del Vice, prima di ammalarsi di cancro. Quella vita ignara di sé, "vera morte», "vero diavolo», che ci rende ciechi rispetto alle ragioni profonde del vivere, quelle ragioni che ormai il Vice fa proprie vivendo, appunto, "per la morte», nel segno e nella luce della morte. ${ }^{50}$

Refuerza esta consideración de Onofri el hecho de que, precisamente tras la reflexión del protagonista frente al grabado, tiene lugar la conversación entre el Vice y su jefe, en la que aquel accede a la recomendación de tomarse una excedencia, que simbólicamente sería, por tanto, el abandono definitivo de una obtusa condición de salud.

De manera totalmente opuesta interpreta ese pasaje de Il cavaliere e la morte Giuseppe Traina, ${ }^{51}$ para el cual la figura a lomos del caballo, pagada de sí misma y que es la verdadera muerte y el diablo, representa a Cesare Auris-

48. Cfr. Vincenzo Paladino, «L'ultimo Sciascia. Il senso del limite», Critica Letteraria, XVIII, 1990 , n. 69, p. 721.

49. En una recensión aparecida tras la publicación de la novela, Alberto Moravia señalaba que el carácter imperturbable del caballero frente a la muerte y el diablo encuentra eco en «'eroismo stoico e disperato» del Vice, que no desiste en su indagación pese a los obstáculos que se le presentan. Vincenzo Consolo, por su parte, ha subrayado el paralelismo entre la situación que atraviesa la figura incisa por Durero y la que experimentó el escritor siciliano en sus últimos años de vida. Cfr. Alberto MORAVIA, "Il cavaliere di Sciascia», Corriere della Sera, 2 de enero de 1989; y Vincenzo CONSOLO, "La conversazione interrotta", en VV.AA., La Sicilia, il suo cuore. Omaggio a Leonardo Sciascia, Racalmuto-Palermo: Fondazione Leonardo Sciascia-Fondazione G. Whitaker, 1992, p. 38.

50. Massimo OnOfrI, Storia di Sciascia, Roma-Bari: Laterza, 2004, p. 274.

51. Cfr. Giuseppe TraINA, "L'ars moriendi di Leonardo Sciascia: Il cavaliere e la morte», Siculorum Gymnasium, XLIV, 1991, n. 1-2, p. 183-199. 
pa. Él es responsable del asesinato de Sandoz, pero se sabe inmune a la investigación policial. Su carácter altivo y desdeñoso, tal y como es descrito en el primer capítulo, según Traina encuentra su paralelismo en la imperturbabilidad del caballero. Más aún, éste aparece de perfil en el grabado y, de igual modo, Aurispa «è un uomo talmente freddo, che mi pare esista soltanto di profilo, come su una moneta, sulle monete», 52 según la descripción de la señora De Matis.

No es extraño, por lo demás, que exista esta dualidad interpretativa, puesto que el propio jinete dureriano, junto con la ya señalada de Panofsky, suscitó otras opiniones divergentes. ${ }^{53}$ En relación con Il cavaliere e la morte, creemos que la interpretación más convincente y completa de este elemento simbólico proviene de Ernestina Pellegrini (como ha reconocido posteriormente el propio Traina).${ }^{54}$ En su ensayo "Alcune idee della morte in Sciascia», a propósito del grabado de Durero, Ernestina Pellegrini indica

La sovrapposizione, nella stessa immagine del soldato, delle due valenze simboliche: da un lato la forza sempre viva e indomabile di chi va alla ricerca della verità (è lo spazio dell'utopia ma anche dell'alto valore morale di alcuni personaggi sciasciani), dall'altro il male storicamente vincente, bardato e nascosto sotto tutte le sue armi. ${ }^{55}$

La figura del caballero, por tanto, condensa en sí misma tanto al Vice como a Aurispa. No representa tan sólo a uno o a otro, como defendían previamente Massimo Onofri y Giuseppe Traina. El protagonista, en su meditación frente al grabado, comprende que el caballero acorazado no es solamente el espejo de sí mismo de camino a la inalcanzable ciudadela de la verdad ("Cristo? Savonarola? Ma no, ma no»); sino que se ha convertido también en la proyección de Aurispa, de su silueta de hombre acorazado por el Poder y la mentira que lleva consigo «la vera morte, il vero diavolo».

La validez de la lectura interpretativa de Pellegrini no radica tanto en la síntesis dialéctica de las propuestas anteriores como en la confirmación de que en la escritura sciasciana la coincidencia de los extremos es un motivo recurrente. Ya entre Rogas y Cres, el investigador y el asesino investigado de Il con-

52. Leonardo SCIASCIA, Il cavaliere e la morte, cit., p. 429.

53. «Este caballero misterioso ha provocado una dualidad de interpretaciones. En un principio se le vió [sic] como un aliado de la muerte, como un personaje de la danza macabra. En las posteriores interpretaciones, encabezadas por Wölfflin y Panofsky, aparece como el caballero cristiano descrito por Erasmo en su Enchiridion Militis Christiani (Manual del caballero cristiano). En efecto, en el Diario de viaje de 1520-21, Durero se refiere a él como Der Reiter (El caballero) y en otro escrito del mismo diario hace alusión a la obra de Erasmo con gran admiración", Concha Huidobro, op. cit., p. 36-37.

54. Cfr. Giuseppe Traina, In un destino di verità. Ipotesi su Sciascia, Milano: La Vita Felice, 1999 , p. 138.

55. Ernestina PELLEGrinI, "Alcune idee della morte in Sciascia», en Italo MEREU (ed.), La morte come pena in Leonardo Sciascia. Da "Porte aperte» all'abolizione della pena di morte, "Quaderni Leonardo Sciascia», n. 2, Milano: La Vita Felice, 1997, p. 82. 
testo (1971), la identificación entre uno y otro llegaba a ser por un momento como la de quien se mira en un espejo. ${ }^{56}$ En la última novela de Sciascia, Una storia semplice (1989), será un gesto involuntario del inspector lo que revele su culpabilidad en el caso que aparenta investigar, como reflejo de su identidad escindida entre ser contemporáneamente perseguidor y perseguido. ${ }^{57}$ Aunque no alcanza esta identificación plena, el juego entre los contrarios también determina la relación entre Bellodi y Don Mariano (Il giorno della civetta, 1961), el impostor abad Vella y el revolucionario Di Blasi (Il consiglio d'Egitto, 1963) o entre el pintor y Don Gaetano en Todo modo (1974).

En este aspecto, cabe identificar como fuente de inspiración e influencia el cuento de Borges Los teólogos, que sin duda Sciascia conocía ${ }^{58}$ y que termina con la toma de conciencia de uno de los protagonistas, Aureliano, de que frente a Dios «él y Juan de Panonia (el ortodoxo y el hereje, el aborrecedor y el aborrecido, el acusador y la víctima) formaban una sola persona». ${ }^{59}$ En definitiva, una cuestión de reversibilidad entre los extremos que engendra - puesto que una cosa puede ser ella misma y su contraria - una realidad paradójica, muy próxima, por lo demás, al drama identitario pirandelliano, cuya presencia atraviesa toda la producción literaria de Sciascia. ${ }^{60}$

Para terminar con la comparación que establecíamos entre la écfrasis crítica y la écfrasis literaria cabe concluir que, frente al análisis iconográfico que Panofsky como historiador del arte defiende con apoyo documental e intención objetiva, el protagonista del giallo sciasciano realiza una meditación personal e íntima, filtrada por su bagaje cultural y literario y por una situación vital que, lejos de ver en la obra dureriana una exaltación de la fe cristiana, la percibe como un memento mori en el que no encuentra consuelo sobrenatural.

56. Cuando Rogas abandona la casa del presidente Riches, se cruza con Cres en el ascensor. Su identificación con el asesino le lleva —incumpliendo su deber-a dejarlo escapar, a sabiendas de que Cres se dirige a acabar con Riches: "Come un sonnambulo si ritrovò dentro l'ascensore; e nel rapido aprirsi dei battenti, nell'atrio, ebbe per un momento la sensazione di trovarsi di fronte a uno specchio. Solo che nello specchio c'era un altro (...) Rogas controllò i propri istinti: senza eccesivo sforzo, bisogna dirlo a suo onore o disonore (a piacer vostro)», Leonardo SCIASCIA, Il contesto, en Opere (1971-1983), cit., p. 76.

57. Al visitar el lugar del crimen junto al sargento Lagandara, el inspector enciende instintivamente un interruptor que estaba escondido, demostrando así que conocía la casa abandonada donde apareció el cadáver de Rocella. El sargento comenta este hecho con un amigo de la víctima: "Io avevo consumato un'intera scatola di frammiferi, cercando quell’interruttore; gli altri erano poi venuti a cercarlo con lampadine tascabili... E lui invece l'ha trovato subito a colpo sicuro". "Incredibile errore, da parte sua" disse il professore. "Ma come ha potuto farlo, che cosa gli è accaduto in quel momento?" "Forse un fenomeno di improvviso sdoppiamento: in quel momento è diventato il poliziotto che dava la caccia a se stesso". Ed enigmaticamente, come parlando tra sé, aggiunse: "Pirandello" ", Leonardo SCIASCIA, Una storia semplice, en Opere (1984-1989), cit., p. 756.

58. Cfr. Leonardo SCIASCIA, 1912+1, en Opere (1984-1989), cit., p. 316-317.

59. Jorge Luis BorGES, El aleph, Madrid: Alianza, 1978, p. 48.

60. Cfr. Pietro Milone, L'udienza. Sciascia scrittore e critico pirandelliano, Roma: Vecchiarelli, 2002. 
Por todo ello, la relevancia que adquiere la interpretación del grabado por parte del Vice está, más que en el valor de su original propuesta crítica, en la condición de instrumento imprescindible para su propia caracterización, a través del cual el lector de Il cavaliere e la morte conoce el estado de ánimo del protagonista. Por tanto, podemos afirmar con Michel Riffaterre que

en modo alguno se puede definir la écfrasis literaria como una lectura, pues lo que descifra en primer lugar no es el cuadro sino a su espectador. Es la interpretación del espectador (del autor) lo que dicta la descripción, y no a la inversa. En lugar de copiar el cuadro transcribiendo en palabras el dibujo y los colores del pintor, la écfrasis lo impregna y lo tiñe con una proyección del escritor - o más bien del texto escrito sobre el texto visual. No hay imitación sino intertextualidad, interpretación del texto del pintor y del intertexto del escritor (...) Si la écfrasis es un enunciado para el historiador y para el crítico de arte, para el escritor es una enunciación. ${ }^{61}$

Hay un último aspecto que conviene considerar al examinar la intertextualidad interartística en Il cavaliere e la morte y es la relación indirecta que se establece entre ésta y otras obras literarias que también han ejercido la écfrasis sobre el mismo grabado de Durero. En el caso de la novela policiaca La sospecha, publicada en 1953 por Friedrich Dürrenmatt, es muy probable que sea una intertextualidad consciente y que, por tanto, habría que encuadrar en el tipo de alusiones implícitas que en el giallo se produce con obras como I promessi sposi o La broma de Kundera. Apoyan esta hipótesis no sólo las importantes similitudes argumentales que presenta el giallo sciasciano respecto a La sospe$c h a$, sino también el hecho de que al año siguiente una frase de Dürrenmatt fuera escogida como epígrafe de Una storia semplice, ${ }^{62}$ indicando así la estima concedida por Sciascia al novelista suizo, al menos en sus últimos años de vida.

Como el Vice, también Bärlach, el protagonista de La sospecha, es un culto comisario de policía, con muchos años de oficio y que está gravemente enfermo; condición que le provoca «un deseo tenaz e indomable de permanecer en este mundo y luchar por otro mejor, sí, luchar incluso con su lamentable cuerpo que el cáncer seguía devorando ávida e inconteniblemente, y al que sólo daban un año de vida como máximo». ${ }^{63}$ La relación que mantiene con su jefe, marcada por la confianza y no exenta de ironías, se asemeja a la que se establece entre el Vice y el Capo. Y en la habitación de la clínica en la que está hospitalizado - a la que acude para investigar si el director es un antiguo criminal nazi que se daba por muerto-, Bärlach pide que coloquen una reproducción de El caballero, la muerte y el diablo, frente al que experimenta una

61. Michel Riffaterre, op. cit., p. 174.

62. «Ancora una volta voglio scandagliare scrupolosamente le possibilità che forse ancora restano alla giustizia. dürrenmatt, Giustizia», L. SCIASCIA, Una storia semplice, cit., p. 731. Sobre las similitudes entre el universo poético del escritor suizo y el del siciliano cfr. Giuseppe Traina, Leonardo Sciascia, op. cit., p. 101-102, s.v. Dürrenmatt, Friedrich.

63. Friedrich DürrenmatT, La sospecha, Barcelona: Tusquets, 1996, p. 60. 
identificación vital análoga a la que se deduce de las reflexiones que el Vice desarrolla a solas en su despacho.

Estos elementos comunes -importantes tanto en la caracterización del personaje protagonista como en el orden temático y simbólico- permiten pensar que, aunque no lo hiciera explícito por la vía de la intertextualidad, Sciascia quería aludir, acaso a modo de homenaje, a la novela de Dürrenmatt.

Es menos probable que el escritor siciliano conociera, por la escasa difusión que alcanzó el volumen, las Variaciones sobre un tema de Durero que Alberto Manguel compiló en 1968, tras proponer a diversos escritores argentinos que escribieran algún relato o poema tomando aquel grabado como fuente de inspiración. ${ }^{64}$ Aunque tampoco se puede descartar que hubiese leído algunos de los textos allí reunidos, pues varios de sus autores incluyeron posteriormente su colaboración en obras propias.

Así procedió Borges con el soneto que había escrito, al que añadió otro para conformar Dos versiones de "Ritter, Tod und Teufel", que formaron parte al año siguiente de su poemario Elogio de la sombra. En el primero de ellos centra la atención en la escena representada por el artista alemán; mientras que en el segundo el escritor, ya septuagenario, se proyecta y compara con la figura del caballero para lamentar, por contraste con ese jinete «imperturbable, imaginario, eterno", la fugacidad de su propia vida. ${ }^{65}$ Puesto que Borges fue perdiendo gradualmente la vista hasta quedar ciego a principios de los años cincuenta, en su ejercicio de écfrasis tuvo que operar necesariamente el recuerdo y, por tanto, debía de conocer muy bien el grabado de Durero antes de que en 1968 Manguel le pidiera que escribiera sobre él. Y efectivamente, su pasión por ese "perdurable sueño de Durero», del que tenía una reproducción en su dormitorio, ${ }^{66}$ se remonta a los años de infancia y juventud, como se deduce de estos versos de Yesterdays, incluido en La cifra, el penúltimo poemario del escritor:
Soy cada instante de mi largo tiempo,
cada noche de insomnio escrupuloso, cada separación y cada víspera.
Soy la errónea memoria de un grabado
que hay en la habitación y que mis ojos,

64. Cfr. Alberto Manguel, Variaciones sobre un tema de Durero, Buenos Aires: Galerna, 1968.

65. «Los caminos son dos. El de aquel hombre / De hierro y de soberbia, y que cabalga, / Firme en su fe, por la dudosa selva / Del mundo, entre las befas y la danza / Inmóvil del Demonio y de la Muerte, / Y el otro, el breve, el mío (...) / A mí, no al paladín, exhorta el blanco / Anciano coronado de sinuosas / Serpientes. La clepsidra sucesiva / Mide mi tiempo, no su eterno ahora. / Yo seré la ceniza y la tiniebla», Jorge Luis BORGES, Elogio de la sombra, Buenos Aires: Emecé, 1969, p. 124.

66. «El dormitorio de Borges (...) era lo que los historiadores militares llamarían "espartano”. Una cama de hierro con una colcha blanca sobre la que Beppo a menudo se acurrucaba, una silla, un pequeño escritorio y dos bibliotecas de escasa altura conformaban el único mobiliario. En la pared colgaba un plato de madera con los escudos de armas de los diversos cantones de Suiza y el grabado de Durero "El Caballero, la Muerte y el Diablo”", Alberto Manguel, Con Borges, Madrid: Alianza, 2004, p. 27. 
hoy apagados, vieron claramente:

El Jinete, la Muerte y el Demonio. ${ }^{67}$

También Marco Denevi tomó su contribución a la obra editada por Manguel para incluirla en una edición ampliada de Falsificaciones, un conjunto de cuentos y microrrelatos en los que hacía una lectura moderna e irónica de sucesos protagonizados por personajes históricos o tomados de la historia literaria, artística o bíblica. En El perro de Durero Denevi ofrece una imagen del caballero del grabado similar a la que proponía el Vice, pues pese al cansancio de volver de la guerra está henchido de orgullo, porque ignora que la muerte anida bajo su propia coraza. ${ }^{68}$

Por su parte, en El bosque de Tarcos Silvina Ocampo proponía una recreación más libre de la obra de arte pues, aunque hacía un seguimiento casi textual de las figuras que en ella aparecen, también incorporaba elementos sorpresivos que le daban colorido (flores violetas que caen de los árboles), detalles humorísticos (la muerte que le toma el pulso al jinete como si fuera su médico) y rasgos caricaturescos, como el hecho de que el caballero, "tan presumido como feo», lejos de pensar en "el mundo del heroísmo, las aventuras, las hazañas», repitiese argentinismos incoherentes. ${ }^{69}$

En definitiva, y volviendo a Il cavaliere e la morte, podemos concluir que buena parte de la fuerza simbólica que para el propio relato se deriva de su carácter ecfrástico proviene de la representación verbal de un motivo iconográfico destacado dentro de la historia del arte, ambiguo y por ello sugerente y rico en posibilidades interpretativas. Por eso ha inspirado diversos textos literarios, con los que el giallo sciasciano comparte mayores o menores semejanzas.

\section{Il cavaliere e la morte como "testamento literario»}

Como hemos señalado en la introducción, Sciascia afirmó con motivo de la publicación de Il cavaliere e la morte que, aunque aún siguiera escribiendo, consideraba esta obra como el auténtico epílogo del conjunto de su producción artística. Por eso, en el giallo que protagoniza el Vice vuelven a comparecer los elementos temáticos y estilísticos esenciales de su universo poético. En el ámbito de la intertextualidad esto se manifiesta en la repetición de referencias intertextuales que ya habían aparecido en novelas y ensayos precedentes del autor y que integran de nuevo un relato sciasciano, ya sea por medio

67. Cfr. Jorge Luis BORGES, La cifra, Madrid: Alianza, 1981.

68. «El caballero no sabe que el perro ha olido alrededor de la armadura el tufo de la muerte y del infierno, pues el perro ya sabe lo que todavía no sabe el caballero, el perro ya sabe que en la ingle del caballero una buba ha empezado a destilar los jugos de la peste negra, y que la Muerte y el Diablo aguardan al caballero al pie de la colina para llevárselo con ellos», Marco DeneVI, Falsificaciones, Buenos Aires: Corregidor, 1996, p. 117.

69. El bosque de Tarcos fue incluido luego por su autora en la recopilación de relatos $Y$ así sucesivamente (1987), cfr. Victoria OCAMPO, Cuentos completos II, Buenos Aires: Emecé, 1999, p. $154-157$. 
del discurso del narrador como a través de intervenciones de aquellos personajes que se erigen como portavoces del pensamiento del escritor siciliano. Así pues, cuando la instancia narrativa recuerda, a propósito de las afinidades que sienten el Vice y otro personaje, el doctor Rieti, que «un toscano del ' 500 aveva detto che i siciliani sono di intelletto secco",${ }^{70}$ podemos saber que está refiriéndose a Giovanni Maria Cecchi porque su «retrato de los sicilianos" ${ }^{71}$ ya había sido extensamente citado por Sciascia en el ensayo "Come si può essere siciliani?», escrito en 1984 como introducción al libro de fotografías de Ferdinando Scianna Il grande libro della Sicilia.

También la descripción de la locuacidad de la señora Zorni incluye una reflexión sobre la estupidez que ya había sido objeto de comentario en otra página sciasciana. Para interpretar correctamente lo que se lee en el giallo:

La signora Zorni. Davvero bella, fino all'insipida perfezione; e di una loquacità che a quella perfezione si accordava: astratta, a testa per aria, divagante nei più celesti e irraggiungibili cieli della stupidità: che sa essere celeste, e anche profonda, come gli intelligenti sanno e, sentendola come seduzione, temono ${ }^{72}$

hay que remitirse al ensayo «Appunto su Bouvard e Pécuchet», en el que, comentando la obra de Flaubert, cita un pasaje de la Nuova Enciclopedia de Alberto Savinio que demuestra que es a él a quien se refiere en Il cavaliere e la morte como una de esas personas inteligentes que sienten la seducción de la estupidez y por eso la temen. ${ }^{73}$

Al explicar la relación amorosa del Vice con «Lei», esa mujer que «aveva desiderato ed amato per anni», el narrador añade que «s'incontravano sempre con gioia - la gioia dei corpi, la sola di cui reciprocamente potevano esser

70. Leonardo SCIASCIA, Il cavaliere e la morte, cit., p. 440.

71. «En el Cinquecento il toscano Giovanni Maria Cecchi dava questo «ritratto» dei siciliani: "Sono avidissimi nel mangiare, ma universalmente sobri nel bere, più che continenti delle cose veneree, e vivono in sì gran gelosia delle loro donne che le tengono molto ristrette, e fanno acerbissime vendette sopra a chi hanno in sospezione. Peccano forte in avarizia, che con vergogna e incomodo loro fanno le spese per il vivere e vestire e ornamento di casa; sono ancora altieri, e dove non è differenza grande di titolo, non si cedono l'uno all'altro; ardenti amici e pessimi inimici, subbietti ad odiarsi, invidiosi e di lingua velenosa, di intelletto secco, atti ad apprendere con facilità varie cose; e in ciascuna loro operazione usano astuzia», Leonardo SCIASCIA, Fatti diversi di storia letteraria e civile, en Opere (1984-1989), cit., p. 520-521. La cursiva es nuestra.

72. Leonardo SCIASCIA, Il cavaliere e la morte, cit., p. 434.

73. "Il fascino che la stupidità esercitava su Flaubert può essere benissimo definito da questo passo di Savinio: "La stupidità, questo inconfessabile amore, esercita su noi un potere ipnotico, una invincibile attiranza. Più volte l'ho sperimentato nel tram, nei luoghi pubblici, al caffe. Sto seduto al caffe, e accanto a me che vado errando nei più inesplorati continenti dell'intelligenza, seggono alcuni sconosciuti. Come avviene di solito, esalano i discorsi di costoro una stupidità ineffabile, ispirata, incantatrice. A poco a poco la mia avventura si offusca, perdo la traccia del mio viaggio solitario, cedo al richiamo primordiale della stupidità, il mio orecchio è pieno della voce della sirena" ", Leonardo SCIASCIA, Cruciverba, en Opere (1971-1983), cit., p. 1102. Cfr. Alberto SAVINIO, op. cit., p. 353-354, s.v. Stupidità. 
certi». ${ }^{74}$ Esta exaltación de la corporalidad como fundamento de la alegría amorosa es idéntica a la ya manifestada en Candido ovvero un sogno fatto in Sicilia, donde el abandono del protagonista por parte de su novia es interpretado por aquel desde la misma perspectiva:

Che Paola se ne fosse andata significava una sola cosa, per lui: che qualcosa era accaduto tra loro che aveva spezzato l'armonia del vivere insieme, la gioia dei loro corpi (...) Si erano incontrati nella verità dei loro corpi, in quella gioiosa verità erano stati assieme. Poi, forse, il corpo di Paola aveva ceduto all'anima. All'anima immortale, all'anima sentimentale, all'anima bella: ed ecco che la gioiosa verità del corpo le si era appannata, le si era stravolta; era diventata un bene inferiore. ${ }^{75}$

Es una concepción y una terminología que - como revela Sciascia en Nero su nero- ${ }^{76}$ proviene de Michel Foucault y cuya invocación, aplicada tanto para el Vice como para Candido en su resistencia frente a los abusos del poder, puede entenderse en el sentido de que "la vera alternativa al sistema dell'alienazione non sta nell'ideologia, ma nella vita stessa, nella "corporalità", nella fisicità dell' esistenza». ${ }^{77}$

No solamente a través del narrador emplea Sciascia esta modalidad de citación literaria, sino que también por boca del Vice y de la señora De Matis el escritor siciliano retoma algunas consideraciones que no son nuevas dentro del conjunto de su obra.

Así se constata al menos en dos ejemplos. Después de descubrir que ambos comparten un origen siciliano, el protagonista le dice a ella: "Non so se lei conosce quella pagina di Lawrence sul Mastro don Gesualdo di Verga. Ad un certo punto dice: ma Gesualdo è siciliano, e qui salta fuori la difficoltà...". ${ }^{78}$ Sciascia ya había citado «aquella página de Lawrence» en el ensayo "Come si può essere siciliani?»:

Pur parlando di Verga, e del romanzo di cui è protagonista mastro don Gesualdo, ad un certo punto David Herbert Lawrence dice: "Gesualdo è un uomo comune, dotato di energia eccezionale. Tal è, naturalmente, nell’intenzione. Ma egli è siciliano. E qui salta fuori la difficoltà». La difficoltà: non si poteva dir meglio, e con una sola parola (ma è giusto segnalare, tra le cose più acute che siano state

74. Leonardo SCIASCIA, Il cavaliere e la morte, cit., p. 456-457.

75. Leonardo SCIASCIA, Candido, en Opere (1971-1983), cit., p. 431.

76. «Finora, insomma, la storia della sessualità è stata fatta attraverso il sesso che parla. Foucault opera uno spostamento radicale: la vede attraverso il potere che lo fa parlare (...) C'è dunque un potere, al di qua o al di là del potere costituito, che può far parlare il sesso senza delirio, senza follia, secondo quella che Foucault chiama "la gioia dei corpi". Ed è il potere della ragione», Leonardo SCIASCIA, Nero su nero, cit., p. 787. Cfr. Michel FOUCAULT, Historia de la sexualidad, 3 vols., Madrid: Siglo XXI de España Editores, 2005.

77. Romano LUPERINI, «Sciascia, la naturalità e il razionalismo», Belfagor, XXXIII, 1978, fasc. 1, no 1 , p. 107.

78. Leonardo SCIASCIA, Il cavaliere e la morte, cit., p. 432. 
scritte su Verga e sulla Sicilia, l'intero saggio di Lawrence). Sicché alla domanda "Come si può essere siciliano?», un siciliano può rispondere: "Con difficoltà». ${ }^{79}$

Avanzada la conversación, que se desvía progresivamente de la investigación sobre el asesinato de Sandoz para acercarse, por medio de las citas literarias, a un tono más distendido; la señora De Matis confiesa su gusto por la relectura y menciona Las almas muertas de Nikolái Gógol. ${ }^{80}$ De nuevo es una idea que, no ya como intervención de un personaje de ficción sino atribuible como pensamiento propio del autor, Sciascia había desarrollado en ocasiones precedentes. En una entrevista concedida al Corriere della Sera el 19 de julio de 1987 declaraba: «Uno dei piaceri della mia vecchiaia è quello di rileggere: prendo un libro letto a treint'anni, di cui ricordo qualcosa che mi ha affascinato, e riscopro una quantità di cose che non ricordavo più». ${ }^{81}$ Años antes, había publicado también en el Corriere della Sera un ensayo que, con el título «Del rileggere», abordaba la misma cuestión:

Naturalmente, bisogna trovare e scegliere un libro per cui valga la pena che tutta una vita ruoti a rileggerlo come la terra intorno al sole. E qui, per non cadere in una aporia, fermo restando il principio che ogni libro viene riscritto nel momento in cui lo si legge, bisogna riconoscere che ci sono dei libri che in sé e più di altri hanno una tale peculiarità e potenzialità; dei libri in sé capaci di diventare «diversi», di arricchirsi e di arricchire la vita di generazioni di uomini, la vita di collettività umane, la vita dei singoli che li leggono, che li rileggono. La Divina Commedia. Il Don Chisciotte. L'Amleto. L'Henry Brulard. E Le anime morte. ${ }^{82}$

En ese mismo ensayo Sciascia calificaba como tema central de Las almas muertas la corrupción, ${ }^{83}$ de tal manera que a través de su invocación por parte de la señora De Matis también se proyecta esa preeminencia temática sobre el giallo, en coherencia con la situación descrita en el relato policiaco, donde aquella es perceptible no sólo en el orden político, sino también en el moral o incluso en el físico - como la enfermedad sufrida por el Vice-.

En definitiva, esta forma de citación basada en la recurrencia diacrónica de referencias intertextuales, por un lado, concede al contenido de dicha reformulación narrativa (la sicilianidad, la relectura como medio de enriquecer la vida, etcétera) mayor relevancia dentro de la serie de temas que conforman el universo

79. Leonardo SCIASCIA, Fatti diversi di storia lettereria e civile, cit., p. 524. Cfr. David Herbert LAWRENCE, Selected literary criticism, London: Heinemann, 1956, p. 275.

80. «Lei legge molto, vero?... Io non molto, e trovo ormai più gusto nel rileggere: si scoprono cose che alla prima lettura non c'erano... Voglio dire: non c'erano per me... E sa che cosa sto rileggendo? Le anime morte: tutto pieno di tante cose che non c'erano; e chi sa quante altre ne scoprirei tornando a leggerlo tra vent'anni...», Leonardo SCIASCIA, Il cavaliere e la morte, cit., p. 433.

81. Citado en "Cronologia», en Leonardo SCIASCIA, Opere (1956-1971), cit., p. LXVIII.

82. Leonardo SCIASCIA, "Cruciverba», cit., p. 1222.

83. Cfr. Ibid., p. 1224. 
poético del autor; y, por otro, al suceder esto en Il cavaliere e la morte, da al relato un carácter distintivo dentro de la producción de Sciascia, como sumario y, al mismo tiempo, versión perfeccionada del conjunto de su obra.

Más aún, podemos concluir que la variedad y recurrencia de formas de intertextualidad - bien por medio de la instancia narrativa, bien a través de los personajes que se erigen como alter ego de Sciascia; ya sea con obras del propio autor, con obras de otros escritores e incluso de otras disciplinas artísticas - muestra que en la cosmovisión sciasciana el espacio de la literatura y del arte es el ámbito donde la verdad alcanza su plenitud. 